\title{
Article \\ Corona Health-A Study- and Sensor-Based Mobile App Platform Exploring Aspects of the COVID-19 Pandemic
}

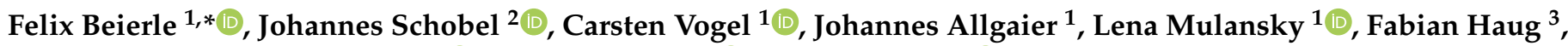 \\ Julian Haug ${ }^{1}$, Winfried Schlee ${ }^{4}{ }^{(}$, Marc Holfelder ${ }^{5}{ }^{(0}$, Michael Stach ${ }^{3}{ }^{-}$, Marc Schickler ${ }^{3}$, Harald Baumeister ${ }^{6}$, \\ Caroline Cohrdes ${ }^{7}{ }^{\mathbb{D}}$, Jürgen Deckert ${ }^{8}$, Lorenz Deserno ${ }^{9}$, Johanna-Sophie Edler ${ }^{7}$, Felizitas A. Eichner ${ }^{1}{ }^{1}$, \\ Helmut Greger ${ }^{10}$, Grit Hein ${ }^{8}$, Peter Heuschmann ${ }^{1}$, Dennis John ${ }^{11}$, Hans A. Kestler ${ }^{12}{ }^{(D}$, Dagmar Krefting ${ }^{13}{ }^{(0)}$, \\ Berthold Langguth ${ }^{4}$, Patrick Meybohm ${ }^{14}{ }^{\oplus}$, Thomas Probst ${ }^{15}{ }^{\circledR}$, Manfred Reichert ${ }^{3}$, Marcel Romanos ${ }^{9}$,

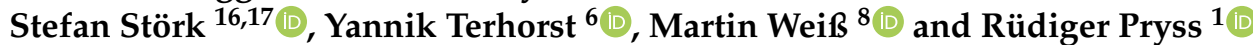

check for

updates

Citation: Beierle, F.; Schobel, J.; Vogel, C.; Allgaier, J.; Mulansky, L.; Haug, F.; Haug, J.; Schlee, W. Holfelder, M.; Stach, M.; et al. Corona Health-A Study- and Sensor-Based Mobile App Platform Exploring Aspects of the COVID-19 Pandemic. Int. J. Environ. Res. Public Health 2021, 18, 7395. https://doi.org/10.3390/ ijerph18147395

Academic Editor: Paul B. Tchounwou

Received: 7 June 2021

Accepted: 6 July 2021

Published: 10 July 2021

Publisher's Note: MDPI stays neutra with regard to jurisdictional claims in published maps and institutional affiliations.

Copyright: (C) 2021 by the authors Licensee MDPI, Basel, Switzerland This article is an open access article distributed under the terms and conditions of the Creative Commons Attribution (CC BY) license (https:// creativecommons.org/licenses/by/ $4.0 /)$
1 Institute of Clinical Epidemiology and Biometry, University of Würzburg, 97080 Würzburg, Germany; carsten.vogel@uni-wuerzburg.de (C.V.); johannes.allgaier@uni-wuerzburg.de (J.A.); lena.mulansky@uni-wuerzburg.de (L.M.); haug_j@ukw.de (J.H.); Eichner_F1@ukw.de (F.A.E.); peter.heuschmann@uni-wuerzburg.de (P.H.); ruediger.pryss@uni-wuerzburg.de (R.P.)

2 DigiHealth Institute, Neu-Ulm University of Applied Sciences, 89231 Neu-Ulm, Germany; johannes.schobel@hnu.de

3 Institute of Databases and Information Systems, Ulm University, 89081 Ulm, Germany; fabian.haug@uni-ulm.de (F.H.); michael.stach@uni-ulm.de (M.S.); marc.schickler@uni-ulm.de (M.S.); manfred.reichert@uni-ulm.de (M.R.)

4 Department of Psychiatry and Psychotherapy, University Regensburg, 93053 Regensburg, Germany; winfried.schlee@gmail.com (W.S.); berthold.langguth@medbo.de (B.L.)

$5 \quad$ LA2 GmbH, 91054 Erlangen, Germany; marc.holfelder@la2.de

6 Department of Clinical Psychology and Psychotherapy, Ulm University, 89081 Ulm, Germany; harald.baumeister@uni-ulm.de (H.B.); yannik.terhorst@uni-ulm.de (Y.T.)

7 Mental Health Research Unit, Department of Epidemiology and Health Monitoring, Robert Koch Institute, 12101 Berlin, Germany; CohrdesC@rki.de (C.C.); EdlerJ@rki.de (J.-S.E.)

8 Department of Psychiatry, Psychosomatics and Psychotherapy, Center of Mental Health, University Hospital Würzburg, 97080 Würzburg, Germany; deckert_j@ukw.de (J.D.); Hein_G@ukw.de (G.H.); weiss_m11@ukw.de (M.W.)

9 Department of Child and Adolescent Psychiatry, University Hospital Würzburg, 97080 Würzburg, Germany; deserno_1@ukw.de (L.D.); romanos_m@ukw.de (M.R.)

10 Service Center Medical Informatics, University Hospital Würzburg, 97080 Würzburg, Germany; greger_h@ukw.de

11 Lutheran University of Applied Sciences Nürnberg, 90429 Nürnberg, Germany; dennis.john@evhn.de

12 Institute of Medical Systems Biology, Ulm University, 89081 Ulm, Germany; hans.kestler@uni-ulm.de

13 Department of Medical Informatics, University Medical Center Göttingen, 37075 Göttingen, Germany; dagmar.krefting@med.uni-goettingen.de

14 Department of Anaesthesiology, Intensive Care, Emergency and Pain Medicine, University Hospital Würzburg, 97080 Würzburg, Germany; meybohm_p@ukw.de

15 Department for Psychotherapy and Biopsychosocial Health, Danube University Krems, 3500 Krems, Austria; thomas.probst@donau-uni.ac.at

16 Comprehensive Heart Failure Center, University and University Hospital Würzburg, 97080 Würzburg, Germany; stoerk_s@ukw.de

17 Department of Internal Medicine I, University Hospital Würzburg, 97080 Würzburg, Germany

* Correspondence: felix.beierle@uni-wuerzburg.de

Abstract: Physical and mental well-being during the COVID-19 pandemic is typically assessed via surveys, which might make it difficult to conduct longitudinal studies and might lead to data suffering from recall bias. Ecological momentary assessment (EMA) driven smartphone apps can help alleviate such issues, allowing for in situ recordings. Implementing such an app is not trivial, necessitates strict regulatory and legal requirements, and requires short development cycles to appropriately react to abrupt changes in the pandemic. Based on an existing app framework, we developed Corona Health, an app that serves as a platform for deploying questionnaire-based studies in combination with recordings of mobile sensors. In this paper, we present the technical details of Corona Health and provide first insights into the collected data. Through collaborative efforts from experts from public health, medicine, psychology, and computer science, we released Corona Health 
publicly on Google Play and the Apple App Store (in July 2020) in eight languages and attracted 7290 installations so far. Currently, five studies related to physical and mental well-being are deployed and 17,241 questionnaires have been filled out. Corona Health proves to be a viable tool for conducting research related to the COVID-19 pandemic and can serve as a blueprint for future EMA-based studies. The data we collected will substantially improve our knowledge on mental and physical health states, traits and trajectories as well as its risk and protective factors over the course of the COVID-19 pandemic and its diverse prevention measures.

Keywords: mobile health; ecological momentary assessment; digital phenotyping; longitudinal studies; mobile crowdsensing

\section{Introduction}

During the COVID-19 pandemic, it is not only the SARS-CoV-2 coronavirus that affects public health, but also the measures taken in trying to contain the spread of the virus, such as lockdowns, home office, and further social distancing measures. Existing studies about the mental health of the population during the COVID-19 pandemic typically use surveys [1-11]. Utilizing smartphone apps for conducting such research has several advantages over web-based surveys: (1) It is more feasible to conduct longitudinal studies and track users' health conditions over time. (2) Through in situ recordings, recall bias can be avoided. (3) Apps allow for mobile sensing to additionally record objective measurements about the user's surroundings and phone usage. (4) Apps offer additional ways of interacting with users, for example, providing feedback that might increase participants' study engagement. However, developing an app for tracking the mental and physical health of the population is challenging. In the case of tracking public health during the COVID-19 pandemic, the three main challenges are: (a) Such an app has to be developed quickly; it has to be robust and easily adaptable; (b) All legal and regulatory aspects have to be complied with; (c) A methodologically sound and valid assessment strategy needs to be implemented that is able to provide new insights on mental and physical health during the COVID-19 pandemic.

The pandemic emerged suddenly and time for developing a sophisticated mobile application was very limited. The typical challenges of mobile software development include frequent updates to the underlying operating systems, a variety of different development alternatives, and a large diversity of devices, sensors, and features. With psychologists, medical and public health experts, software engineers, computer scientists, and privacy experts, we have many stakeholders from a variety of fields whose requirements have to be met. While technical frameworks specifically for mHealth (mobile Health) scenarios exist [12-16], this does not mean that they are off-the-shelf solutions for complex scenarios and requirements from diverse stakeholders. A recent survey on mHealth frameworks listed features regarding multilanguage support, privacy, and customization of data collection as mostly open issues [16]. In addition to that, with the increasing ubiquity of the smartphone and its use for medical purposes, the laws and regulations to be compliant with are increasing $[17,18]$. The European Union recently introduced the EU Medical Device Regulation (MDR), which allows registering an app as a medical product, which was done for our app Corona Health (https: / www.corona-health.net).

The goal of Corona Health is to track mental and physical well-being during the COVID-19 pandemic. The app allows working in an interdisciplinary manner with experts from different fields to deploy different questionnaires and also includes mobile sensing of sensor data and smartphone usage statistics. Corona Health was initiated by the Mental Health Research Unit of the Robert Koch Institute, the German federal agency for public health responsible for disease control and prevention. Technically, Corona Health was built with the TrackYourHealth platform [19], which has been successfully utilized in long-running studies about tinnitus, stress, and diabetes [20-22]. The development time 
of Corona Health was only 3 months, including regulatory and legal aspects as well as approval by the ethics committee of the University of Würzburg. Its finalization within 3 months shows that the TrackYourHealth platform including its established procedures are proper instruments to develop study- and sensor-based mHealth apps. The results presented here may help other researchers working in similar fields and can serve as technical reference for future study results based on Corona Health.

The main contributions of this paper are:

- Introduction of the Corona Health app platform and its technical details;

- Presentation of statistics about data collected with Corona Health from 27 July 2020 to 11 May 2021.

In this paper, we present the app's architecture and its features, illustrate the database, the data exchange between app and backend, detail the study procedure (user journey), and give insights into the mobile sensing features. Special attention is paid to how Corona Health fosters the exchange between computer scientists and healthcare professionals. For this, we present the content pipeline, which is used for multilanguage support, feedback, and deployment of new studies. Additionally, we give a brief introduction to dealing with the medical device regulation. Corona Health was released on Google Play and the Apple App Store on 27 July 2020. At the time of writing, we count 7290 installations and 17,241 filled-out questionnaires overall. We present the app as well as statistics about the collected data.

The paper is structured as follows. In Section 2, relevant background information and related works are discussed. Section 3 introduces the technical details of Corona Health, starting with the user's perspective. Section 4 introduces the data collected so far and provides descriptive statistics, which are then discussed in Section 5.

\section{Background and Related Work}

Ecological Momentary Assessment (EMA) is a research method that focuses on assessing momentary behavior, feelings, or symptoms, typically via self-reports [23,24]. EMA is often applied in psychological research. Using mobile devices for EMA allows for higher validity [25] and filling out a questionnaire in situ helps avoid recall bias [26]. Additionally, mobile devices, typically smartphones, are equipped with several different sensors and can yield statistics about when and how the users interacts with the device. Collecting such context data [27] is called mobile sensing. Generally, we can distinguish between two types of sensing. Participatory sensing focuses on the active collection and sharing of data by users [28]. Here, the user actively decides about the sensing process. Opportunistic sensing, on the other hand, refers to passive sensing with minimal user involvement [29]. In the context of this paper, filling out a questionnaire can be regarded as participatory sensing, and acquiring sensor data such as the user's location can be considered as opportunistic sensing.

Mobile crowdsensing (MCS) refers to the measurement of large-scale phenomena that cannot be measured by a single device [30-32]. This can be, for example, measuring the noise-level across a city, or, as in the context of this paper, measuring the mental and physical well-being of people during the ongoing COVID-19 pandemic. MCS in the healthcare domain can bring its own challenges, such as specific regulations and providing feedback to users [31,33]. The combination of EMA and MCS allows for so-called digital phenotyping [34-39]. Digital phenotyping describes the idea of extending the concept of a phenotype to the behavior of people in relation to digital devices and services, in particular, smartphones. Digital phenotyping research is conducted, for example, in personality science [40,41], for mental health assessments [42] or for predicting mood changes [43].

There are several technical frameworks for the development of MCS apps that support EMA, for example, AWARE [12] or Sensus [13]. In a recent survey paper, Kumar et al. reviewed such frameworks, specifically for mHealth studies and applications [16]. Kumar et al. define three types of stakeholders-researchers, developers, and end users. Only two frameworks support all three of the defined stakeholders. There are further differences between the surveyed frameworks. There is a large range of offered 
features and functionalities, for example, with respect to the types of sensor data that can be collected. Perhaps, due to the effort associated with it, most of the reviewed frameworks are not maintained after release.

As open challenges, the authors of [16] list, among others, a lack of internationalization support. Only one of the reviewed frameworks supports surveys in multiple languages. We suspect that such shortcomings lead to the development of new or specialized frameworks. As another open challenge, Kumar et al. describe the personalization of data collection and the handling of user consent, which in most frameworks have to be implemented by the developers utilizing the framework.

Although users in general are quite willing to share data with researchers [44], especially for COVID-19-related apps, privacy is a major concern [45,46]. Typically, the use of apps for studies has to be approved by institutional review boards, and developers have to comply with local privacy-related laws such as the GDPR (General Data Protection Regulation) in the European Union. On top of that, additional local or regional laws and regulations can bring additional requirements for researchers and developers. Germany introduced regulations about medical devices, which includes strict regulations for apps relating to healthcare. Effectively, these regulations allow apps to be approved as medical products. Additionally, both Google Play and the Apple App Store introduced special requirements for COVID-related apps.

We built Corona Health based on our own framework platform TrackYourHealth, tackling the shortcoming of existing frameworks. We support multiple languages and comply with all local laws, including the German medical product regulations for healthcare-related apps.

\section{Technical Details}

In this section, we present the technical details of Corona Health. Corona Health was built with the TrackYourHealth platform and API $[19,47]$ and consists of a PHP-based backend and two native apps (Android and iOS). The management of content is performed through a content pipeline developed by the team over years. In the future, a web-based application is scheduled to manage the pipeline more conveniently. In the following, we start by describing the contents of Corona Health from a user perspective (Section 3.1). In Section 3.2, we detail the overall architecture and features from a technical perspective. Section 3.3 describes the database schema and what data are recorded. Section 3.4 summarizes how the communication between mobile apps and backend works. Section 3.5 describes the mobile sensing that is conducted in Corona Health. Section 3.6 details how data get into the Corona Health system from a backend perspective, for example, for integrating new studies. This integration of new studies allows for further collaboration with new research partners. We conclude the technical description of Corona Health with a brief overview of how compliance with the medical device regulations in Germany was implemented (Section 3.7). While Corona Health's technical base can serve as a blueprint for similar EMA apps, its concrete implementation and release process cover some aspects specifically related to COVID-19. This includes release requirements such as the strict guidelines set by the app store providers, and this includes features such as the curated coronavirus-related news tab.

\subsection{User Perspective}

Users can download the Corona Health app from Google Play and the Apple App Store for free. The app can be found via the search functionality of the stores, and the app was advertised on Twitter and in newsletters. Figure 1 shows the flow of using Corona Health from a user perspective. The first time the app is used, the app shows the disclaimer (see (1) in Figure 1). Without consent, the app will be closed. After consenting, the user is asked about if he/she wants to allow notifications (2) in Figure 1; iOS shown here). 


\section{Corona Health User Journey}

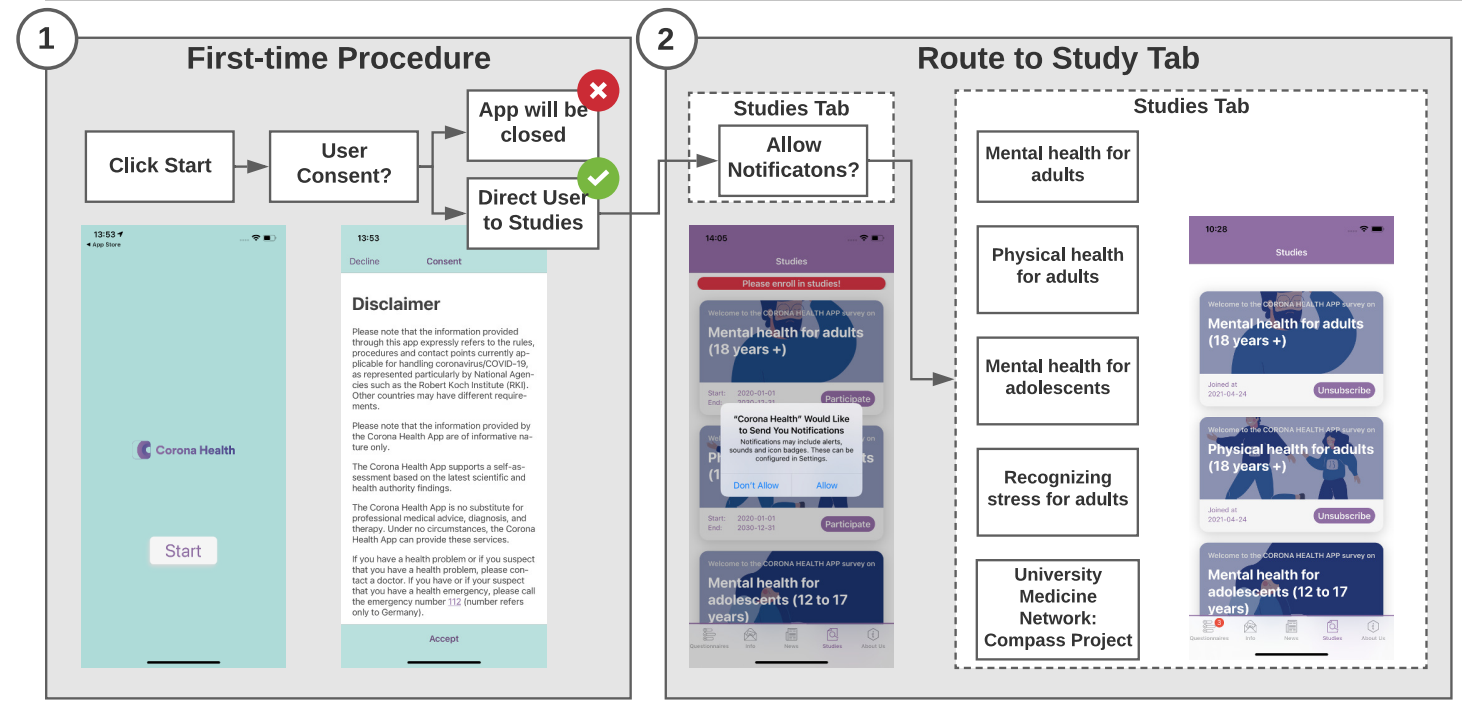

(3)

First time at tab Studies with first questionnaire

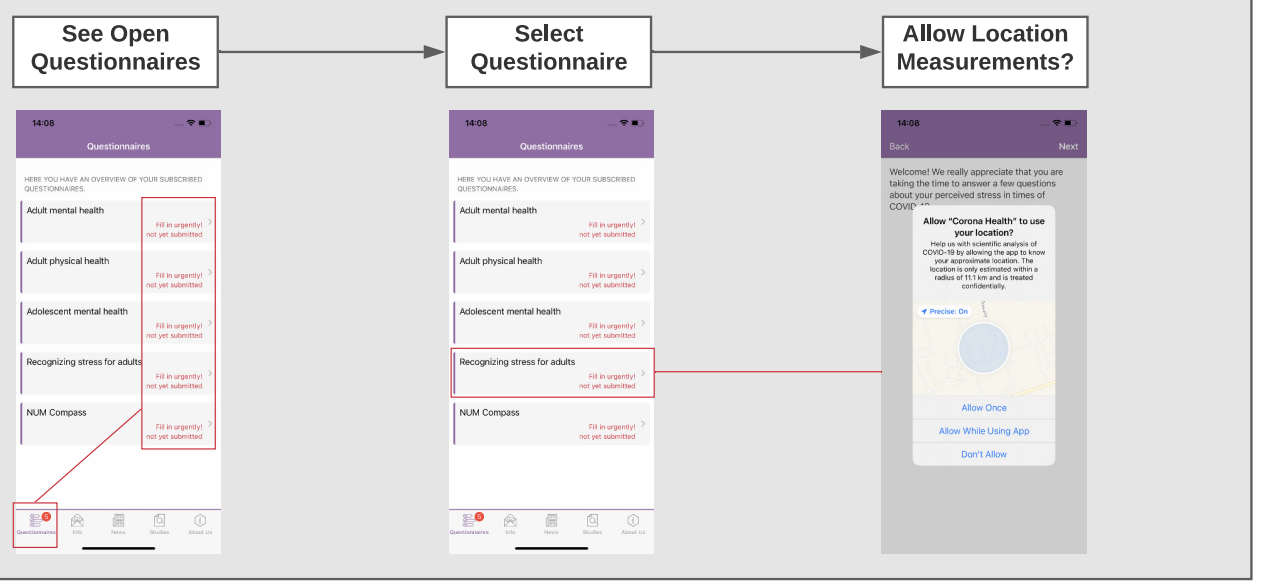

(4) App Usage after Onboarding

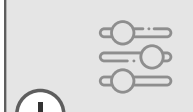

(1)

Questionnaires

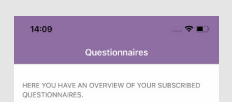

Adult mental ha:

$\mid$ Adu

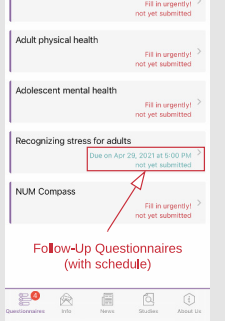

III)

(II)
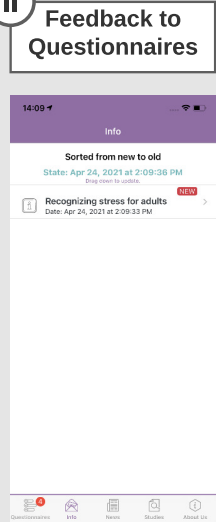

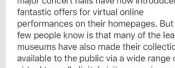

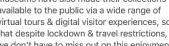

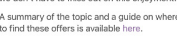
+++ How can we sper
distance? +++
(III) Weekly News

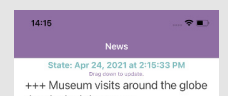

+++ Museum visits aro
despitit lockdonn n++
updated on 14.04.2021 (SG)

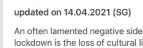

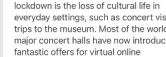

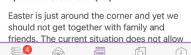

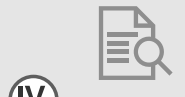

(IV) Study Selection
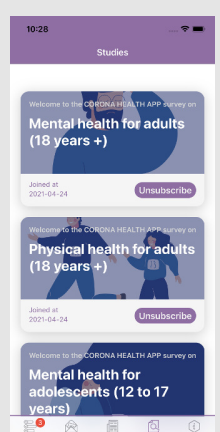

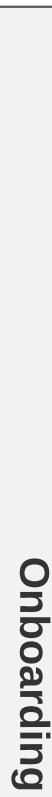

Figure 1. User journey of Corona Health, highlighting the steps for initial start of the app, registration for studies, filling out baseline and follow-up questionnaires. 
The Studies Tab then shows the five currently available studies. Each study that the user selects to participate in contains an initial baseline questionnaire. The user selects each of those in order to open it. At this point, the app asks the user if he/she wants to allow the mobile sensing features to be activated. Point (3) in Figure 1 shows this for iOS (location) and for Android, additionally to location, app usage statistics can be chosen to be shared. We present more details about mobile sensing in Section 3.5. Filling out the baseline questionnaire concludes the onboarding phase of Corona Health.

After onboarding (4) in Figure 1), the user has access to the five main features of the app: Questionnaires, Info, News, Studies, and About Us. The follow-up questionnaires are scheduled by the system. The Info tab contains feedback based on the filled-out questionnaires. The News tab contains weekly news curated by selected members of the provided studies. Through the Studies tab, the user can sign up for additional studies that he/she did not sign up for yet. The About $U s$ tab contains settings, Corona Health team information and all legal policies. Figure 2 shows screenshots of both the Android and the iOS versions of Corona Health.

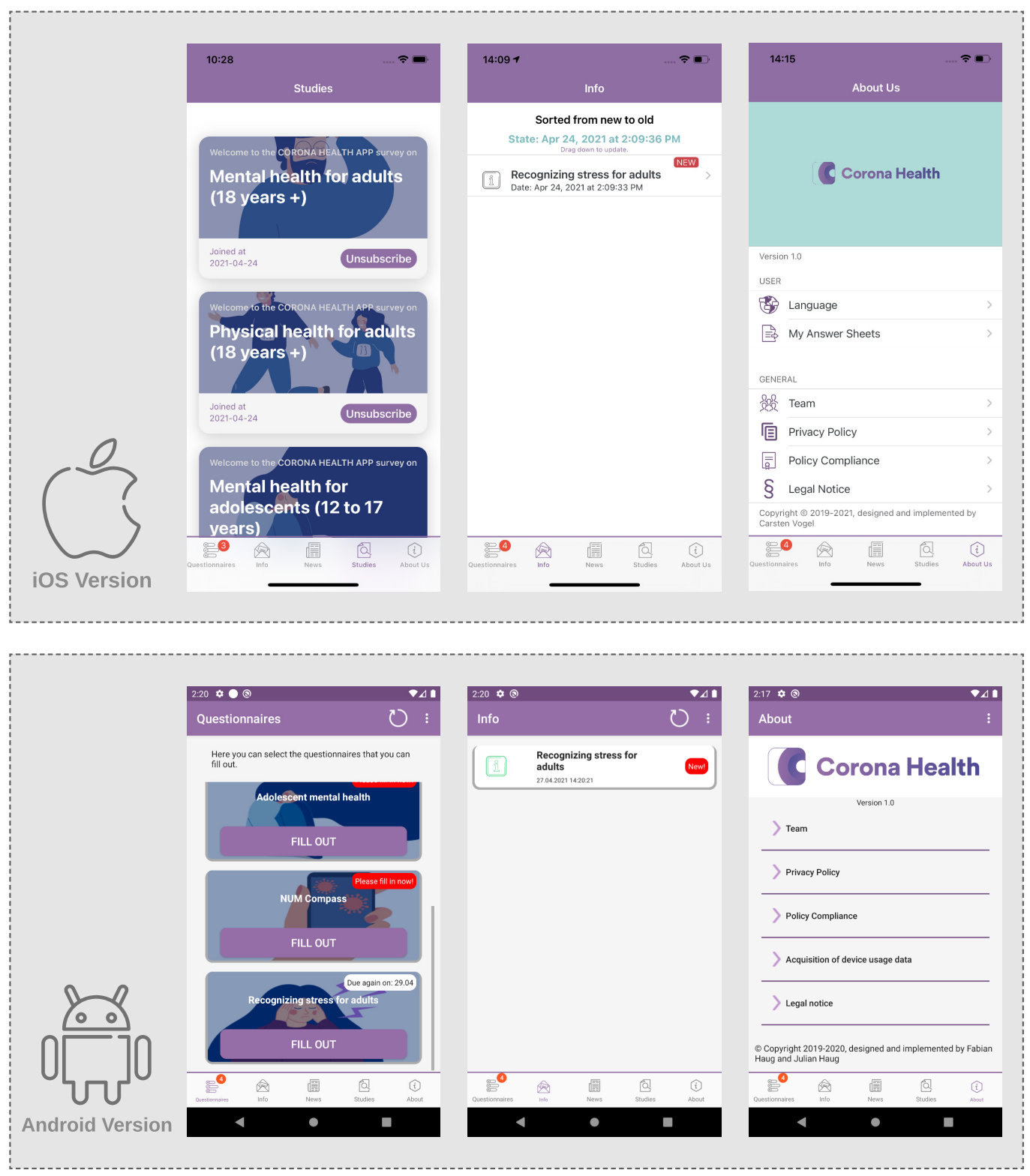

Figure 2. Screenshots of the iOS and Android versions of Corona Health. 
Figure 3 shows the flow for filling out questionnaires, the core feature of Corona Health, focusing on notifications. After the baseline questionnaire, the notification schedule for the selected study is activated. Based on that schedule, notifications will be triggered. Additionally, the user can always fill out additional follow-up questionnaires by manually accessing them in the app.

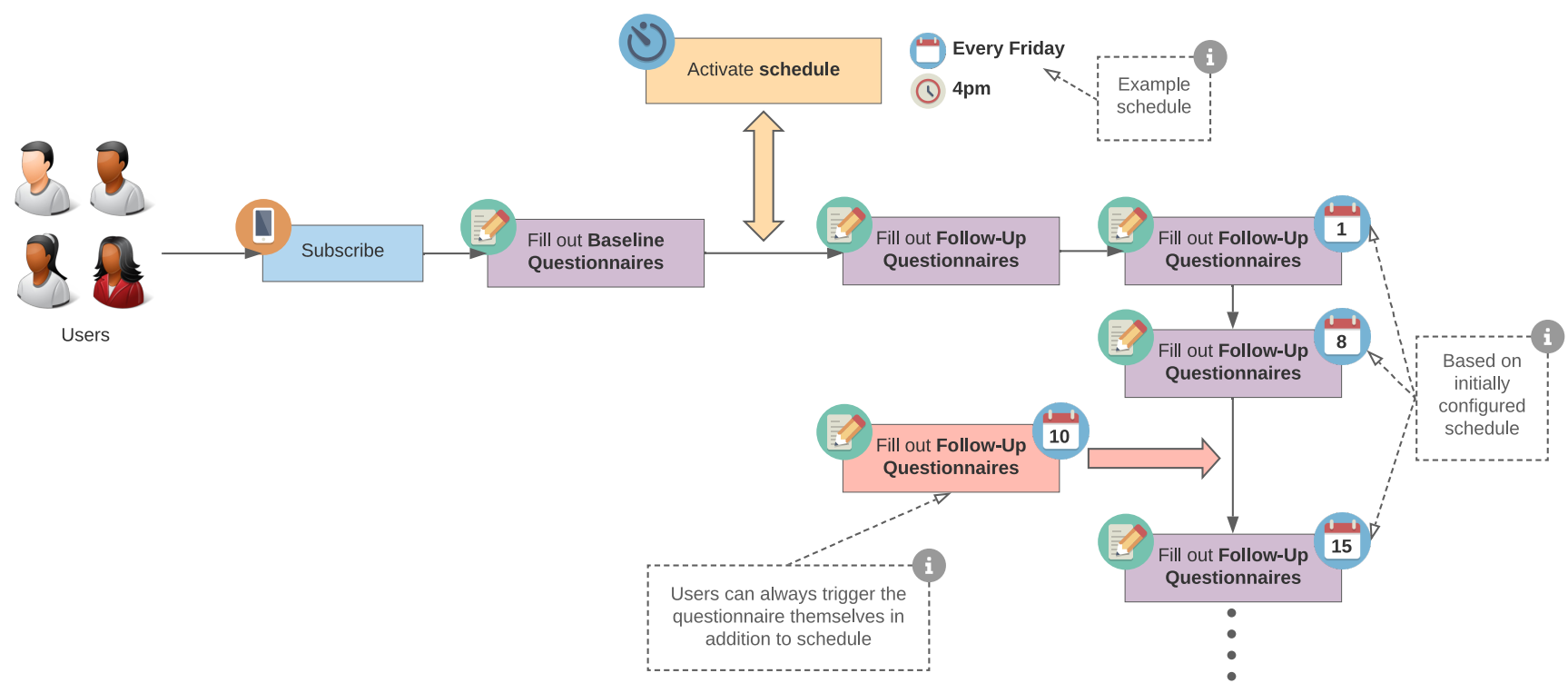

Figure 3. Notification scheduling in Corona Health. After the baseline questionnaire is filled out, the notification schedule for the selected study is activated. Notifications will be triggered based on the given schedule. Additionally, the user can always fill out additional follow-up questionnaires by manually accessing them.

\subsection{App Architecture and Modules}

Figure 4 is a flowchart depicting the processes in the app from a technical perspective. The continuous lines show user interactions and the dashed lines show data interactions in the background. After installation of Corona Health, the app can be started and an anonymous login process is started. We developed the app with offline functionality in mind. If the backend server cannot be reached, the local database temporarily handles the login, storage of questionnaires and mobile sensing data.

Figure 5 shows the core features of Corona Health, divided into backend and mobile device modules. The backend modules are handled by the admin. The user can interact with the modules on the mobile device. The management of the notifications is carried out by the admin.

\subsection{Database Schema}

Corona Health uses a relational database to store and manage both user-generated and operational data. Since data integrity and consistency mechanisms (e.g., constraints and ACID transactions) are already integrated with relational databases, highly interrelated data models are easier to create and maintain with this type of database system. Furthermore, the query language SQL is suitable for complex analytical queries needed for the data evaluation. In order to give insights into the project's database schema, Figure 6 depicts an Entity-Relationship Model (ERM) using Martin's notation. Note that Figure 6 illustrates an excerpt of the entire database schema. The selection represents the core entities containing most of the user-generated data. Due to the multilingual project setup, the database schema contains several entities with translated text data, marked with the red $(T$, which are omitted in the ERM to further simplify the model. 


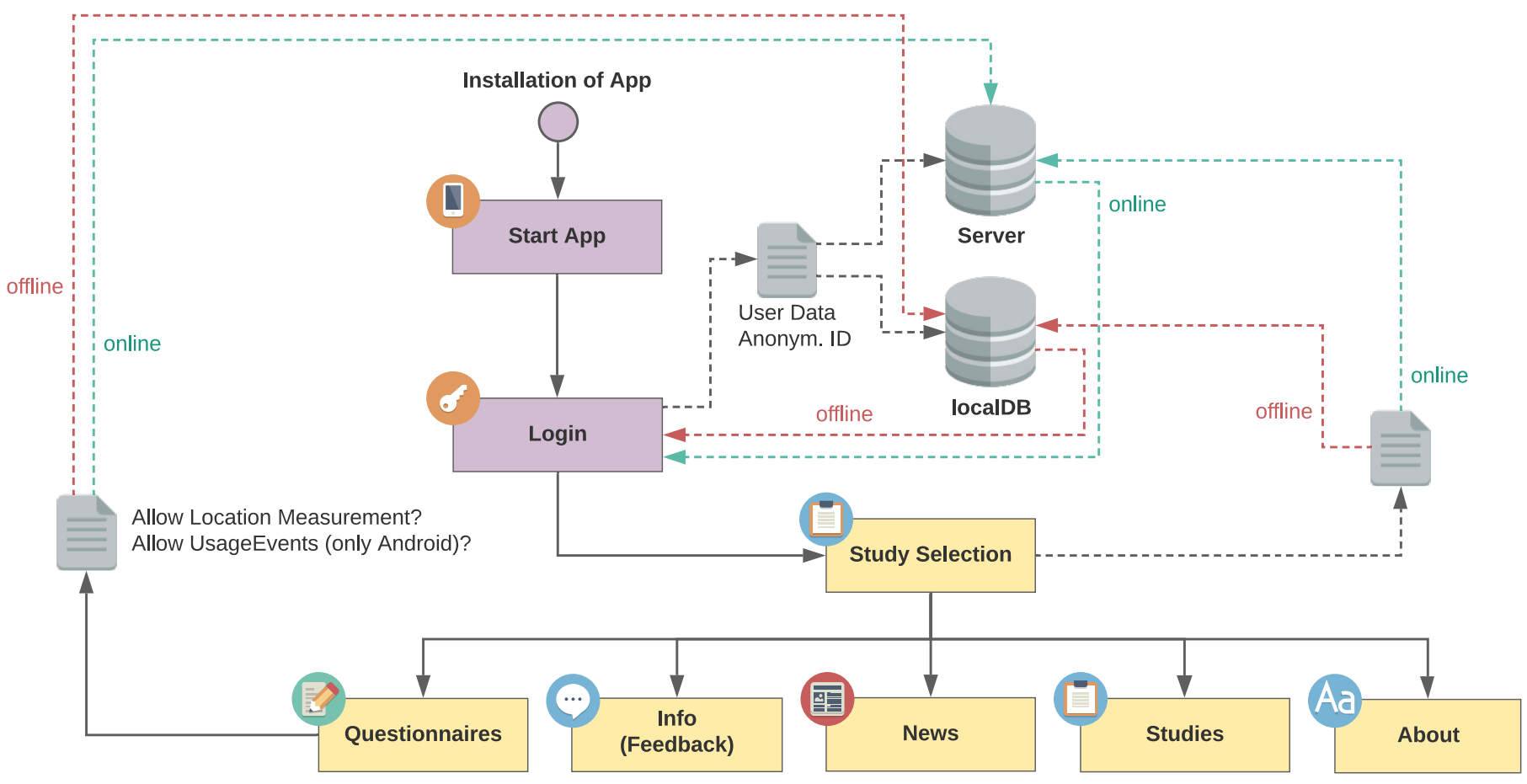

Figure 4. Corona Health processes of the User Journey on a technical level. Continuous lines indicate user interactions and dashed lines indicate background data interactions. After installation, the app can be started and an anonymous login process is started. If the backend server cannot be reached, the local database temporarily handles the login, storage of questionnaires and mobile sensing data.

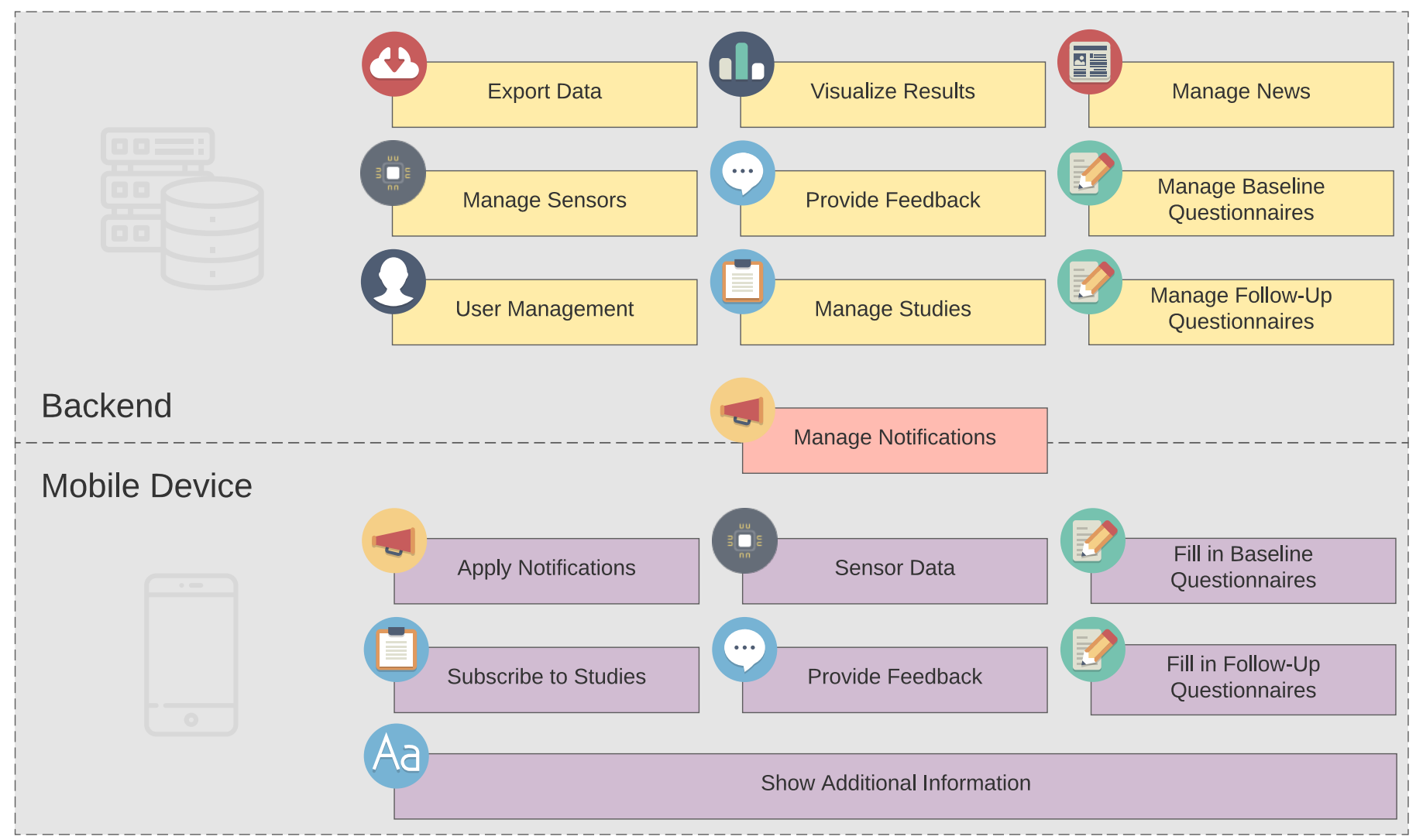

Figure 5. The core modules of Corona Health. 


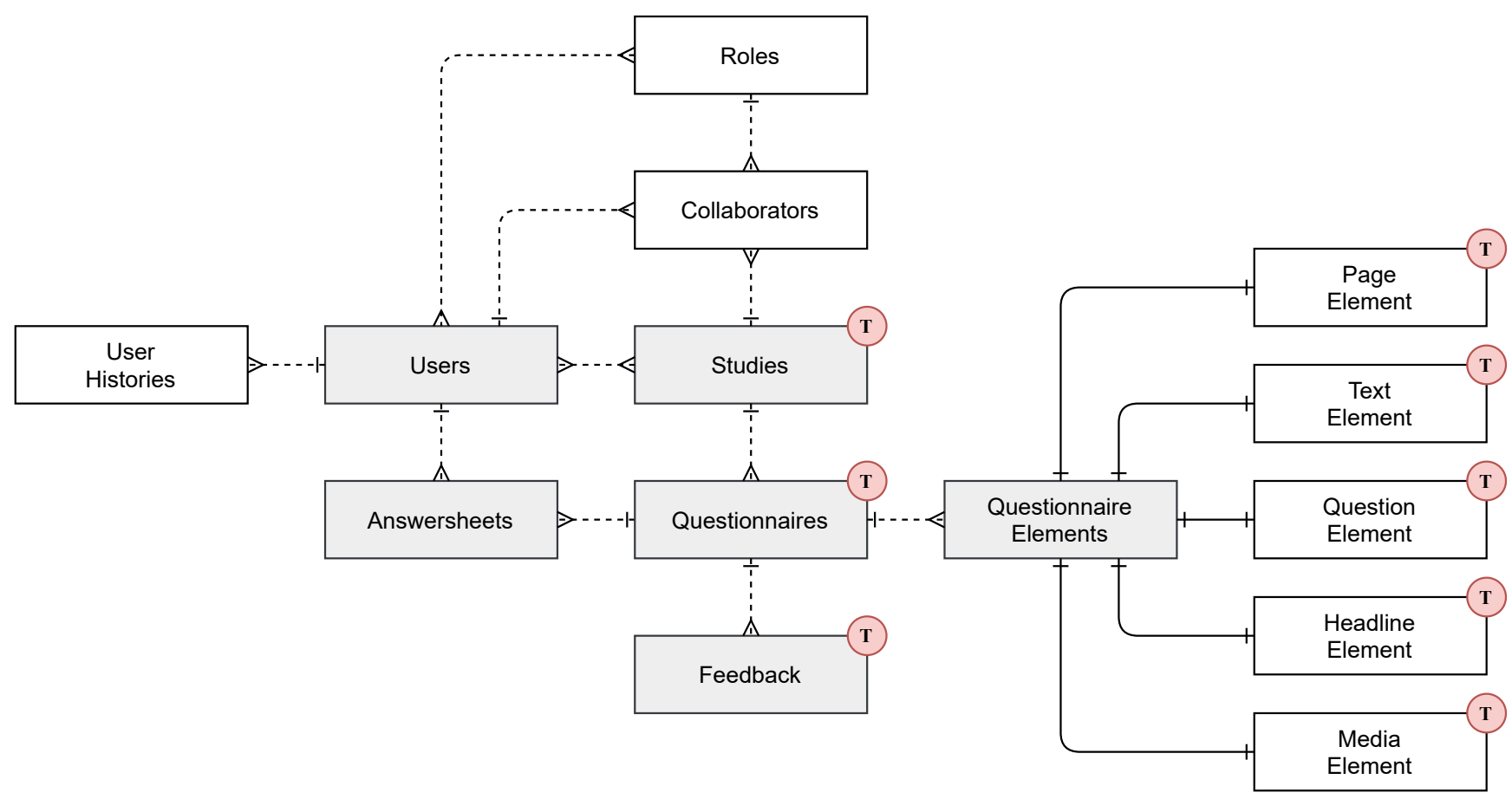

Figure 6. Excerpt of Corona Health's relational database schema. The circled $(T)$ indicates the presence of translated text data.

To describe the data set in this work, a snapshot was extracted on 11 May 2021. Corona Health combines MCS and EMA to collect user data. For this reason, the entity Users constitutes a central and high-related table with 7290 verified users. In addition, the users' actions (e.g., study subscription) are stored in the table User Histories to give insights into the app usage. Currently, there are 4495 subscriptions of 2802 unique users to five multilingual studies. So-called collaborators manage the systems' studies, who are users with additional (role-based) permissions for specific studies.

To collect data in a structured way, one study has one or more questionnaires in the entity of the same name. The latter contains 10 unique questionnaires in each language of the corresponding study. Questionnaires are structured with polymorphic buildings blocks called Questionnaire Elements $(n=1276)$. On average, each questionnaire is structured with 255 elements that can be of the type Page Element $(n=117)$, Text Element $(n=159)$, Question Element $(n=976)$, Headline Element $(n=24)$ or Media Element $(n=0)$. The submitted answers for a questionnaire are serialized and stored in JSON in the entity Answersheets $(n=17,241)$. The latter also stores sensor data (e.g., location) and client device information (e.g., operating system) in the same table. In order to give the user feedback immediately after submission of a questionnaire, such a questionnaire may reference to one or more key-rule pairs that are stored in the entity Feedback $(n=54)$. Rules, evaluated on the client side, can be managed and adjusted dynamically. Feedback translations can also be stored here.

\subsection{Data Exchange}

In the course of developing Corona Health, a RESTful backend service was developed. This backend, in turn, serves as a common API for all clients (i.e., smart mobile devices and web applications). The server application was developed using the well-known Laravel framework and relies on JSON:API (https://jsonapi.org/; last accessed: 15 March 2021) as a specification for building APIs for exchanging data. This specification, in turn, increases productivity by structurally describing request and response formats. JSON:API describes how a client application should request and modify resources and how a server should response to such requests.

The backend server offers routes to access resources from the data model (see Figure 7). In particular, resources denoted in the Design Time part can be accessed by clients. For exam- 
ple, details about Studies or the Questionnaires assigned to Studies can be requested. A Questionnaire, in turn, comprises different Elements, such as Headlines, Texts, or actual Questions to collect data, respectively.

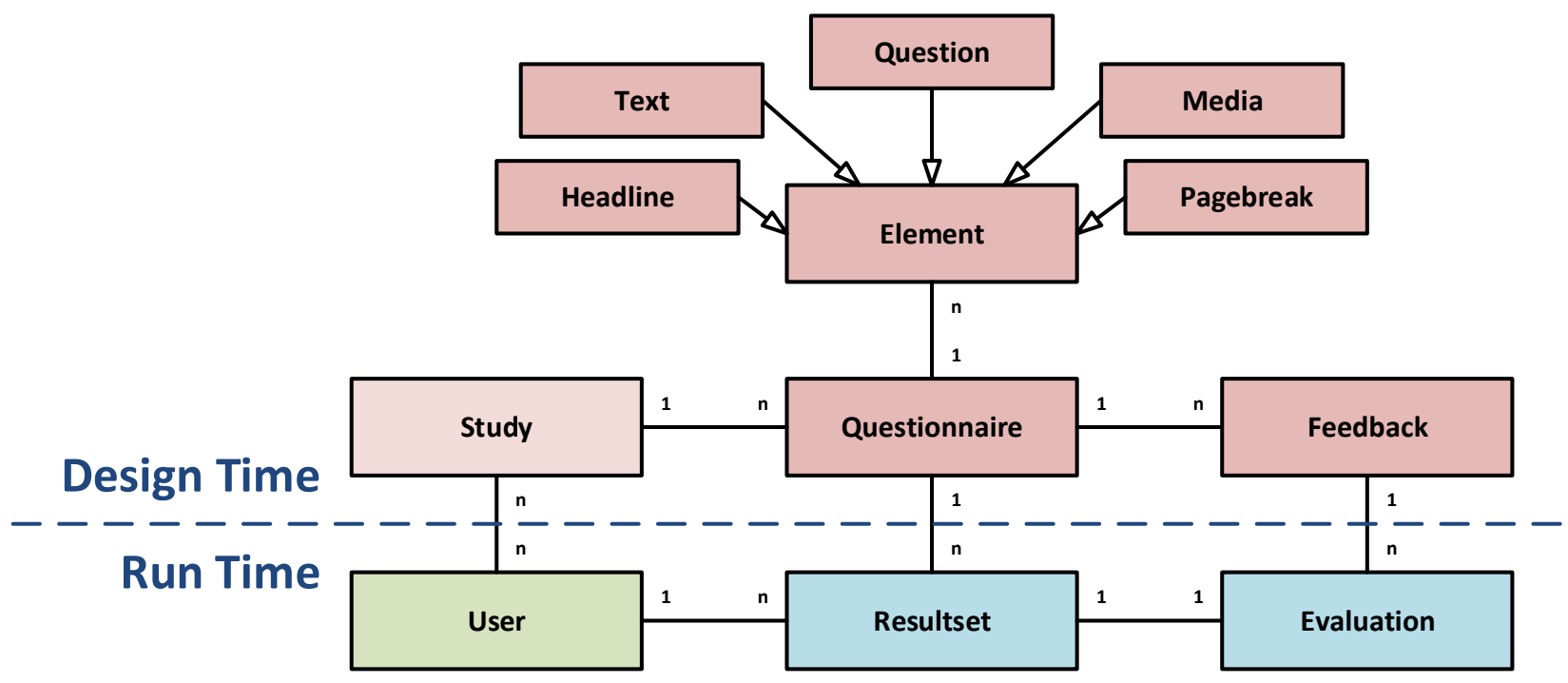

Figure 7. API data model.

Finally, healthcare professionals may define Feedback rules that may be used to automatically evaluate filled-out Questionnaires. Clients, in turn, can submit data via RESTful endpoints for resources described in the Run Time part. In particular, when answering Questionnaires, a Resultset is created. For each Resultset, an Evaluation based on the previously defined Feedback can be requested. In addition to this automated feedback, the API also offers a direct patient-to-doctor communication via a dedicated messaging API (which is not shown in Figure 7).

By its architecture design and the use of JSON:API, the backend service can be easily adapted to future requirements. For example, a psycho-education module was recently implemented and further modules are planned.

\subsection{Mobile Sensing}

Corona Health collects mobile sensing data that are relevant in the context of conducing research related to the physical and mental well-being of the population during the COVID-19 pandemic. The data we collect are device information (Android and iOS), coarse location data during questionnaire fill-out (Android and $\mathrm{iOS}$ ), and aggregated app usage statistics (Android only). All of these data have been shown to be relevant for research. For example, for EMA apps, it has been shown that the used OS should be used as a covariate in analyses [48]. Location data have been shown to have some predictability for depressive mood [49] and positive affect [50]. The averages for app usage statistics have been shown to be different for users with different scores on the Big Five personality trait scales [51].

Each time a questionnaire is filled out, device info, and OS version are stored. If the user gives his/her permission, we also query for and store the current location. The permission for the location is handled by the operating system (Android or iOS). We do not store the raw location, but process the data that we obtain from the OS to make it more coarse grained, and only keep a value with an accuracy of $11.1 \mathrm{~km}$. The level of detail with which we store the mobile sensing data was determined through discussions with all stakeholders, institutional review board, and the relevant laws and regulations.

On Android, if the user gives the relevant permission, it is possible to access the user's app usage history via UsageEvents (https: / / developer.android.com/reference/android/ app/usage/UsageEvents, last accessed: 18 March 2021). The data allow a very detailed look into the users' interactions with his/her smartphone. Before storing data locally and 
sending it to the backend, we aggregate some statistics and only store: (1) Overall daily phone usage; (2) Time periods of activity and inactivity; (3) For some apps: daily sum of usage; timestamps of first and last usage. The apps for which we store some usage statistics are the combination of a fixed list of social media apps and the five most used apps in terms of screen time. Tables 1 and 2 give a detailed overview of the data being collected.

Table 1. Data collected about app usage in Corona Health (Android).

\begin{tabular}{ll}
\hline Field & Comment \\
\hline beginTime & Beginning of observed period \\
\hline endTime & End of observed period \\
\hline collectedAt & Timestamp of observation time \\
\hline apps & List of apps and their usage statistics (see Table 2) \\
\hline top5Apps & Top 5 most used apps and their usage statistics (see Table 2) \\
\hline sleepTimes & $\begin{array}{l}\text { List of tuples with beginning and end timestamps of time windows in which the } \\
\text { device was sleeping for at least one hour (no active screen) }\end{array}$ \\
\hline screenTime & $\begin{array}{l}\text { Contains two lists with daily cummulated time values. List 1: use time of all apps } \\
\text { (screen on with app in visible foreground). List 2: time in which the screen was } \\
\text { active (including no app being used or woken up from a notification). }\end{array}$ \\
\hline
\end{tabular}

Table 2. Values stored for each app that is being recorded (Android).

\begin{tabular}{ll}
\hline Field & Comment \\
\hline packageName & Name of the package \\
\hline completeUseTime & Sum of daily use time \\
\hline completeFGServiceUseTime & $\begin{array}{l}\text { Sum of ForegroundService use time (app is running without being } \\
\text { visible on the screen) }\end{array}$ \\
\hline dailyValues & $\begin{array}{l}\text { List of containers for the following values and timestamps. One } \\
\text { Container per day. }\end{array}$ \\
\hline useTime & Time the app was in the foreground (visible on the screen) \\
\hline lastUseTime & Timestamp of first visible use \\
\hline FGServiceUseTime & Timestamp of the last visible use \\
\hline firstFGServiceUseTime & $\begin{array}{l}\text { Sum of ForegroundService use time (app is running without being } \\
\text { visible) }\end{array}$ \\
\hline lastFGServiceUseTime & Timestamp of first ForeGroundService use \\
\hline
\end{tabular}

\subsection{Content Pipeline}

This section describes the process of bringing the contents of questionnaires into the app. In each of the four stages, experts with different domain knowledge are included. This includes app developers, medical professionals, psychologists, etc. Existing publications also show that such tight communication procedures are highly needed [52]. The entire process for Corona Health, in turn, is shown in Figure 8. In the first stage, the questionnaire is designed in a discussion group. At the same time, information is provided about the technical framework so that it can be incorporated into the questionnaire design. This involves, for example, information on how certain types of questions can be technically represented (e.g., slider, etc.). 


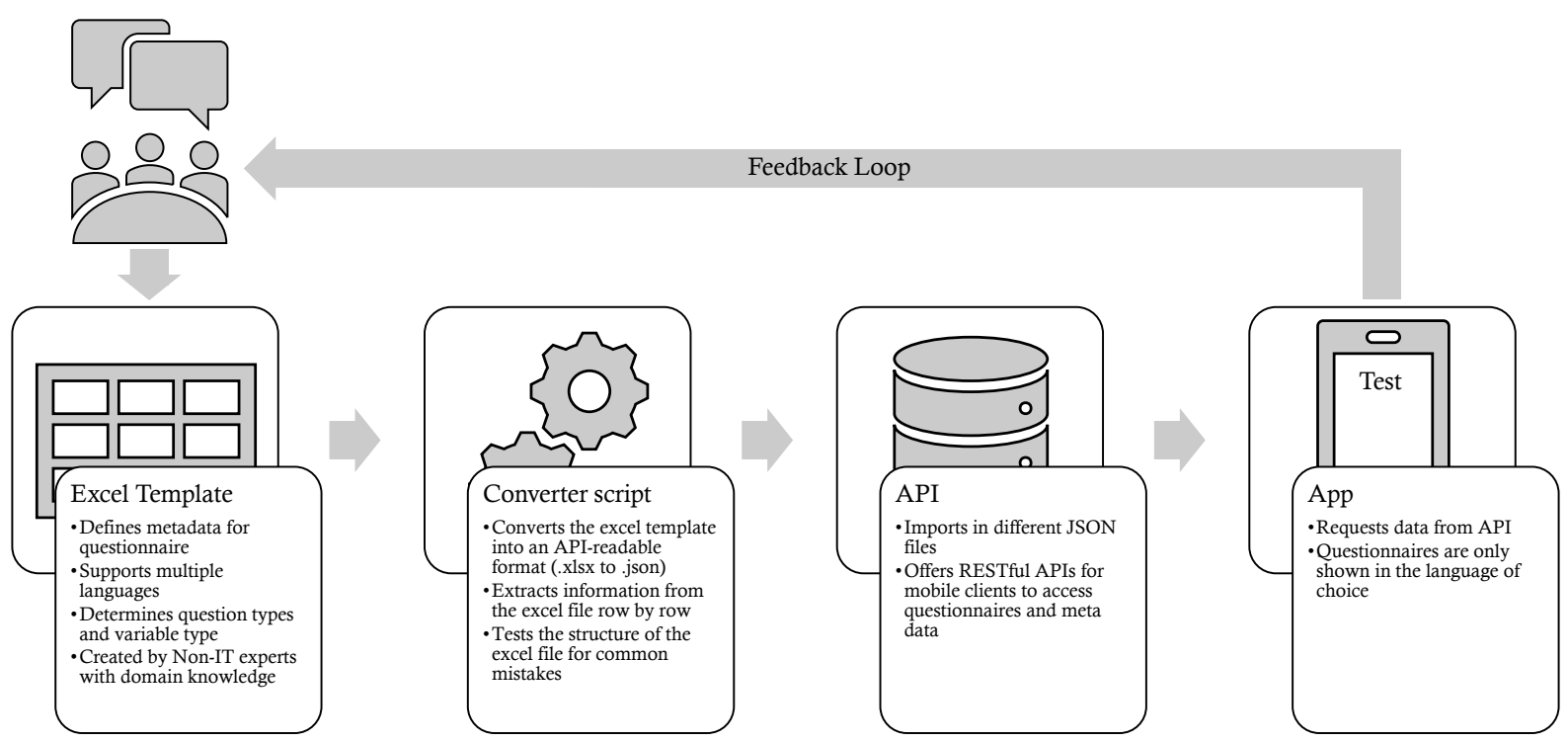

Figure 8. Steps to publish a questionnaire within the mobile application. The automated conversion allows for fast iteration cycles, resulting in a more flexible and end-user driven development process.

In order to create a questionnaire, an Excel template is used. It has two sheets labeled Baseline and FollowUp. This corresponds to the principle of one sheet per questionnaire. In each of the sheets, one line describes one element in the questionnaire. This element, in turn, could be either a headline, a textfield, a pagebreak, or a question. For each question, the creator of the questionnaire has to define which type it has, if it is optional, what the variable name is, and how the answer options should be encoded. Different encodings are highlighted in different colors. The encodings and number of response options must be consistent across all languages. If a certain question is repeated in the follow-up questionnaire, the template obtains all the content of this question using a unique variable name and cross referencing.

The converter script (second step in Figure 8, i.e., the second stage) splits up the Excel sheet into two types of subsets. The first type contains the meta data about the questionnaire; the second type contains the questionnaire itself with one subset per language. Depending on the monitor scaling or version of Excel user, unwanted spaces or line breaks are generated, which then lead to incorrect or unintended display of the questions when called up in the app. For the language subsets, the converter script parses each line and cleans up the strings by removing whitespaces or unintentional line breaks. It finally saves the questionnaire as a $\cdot \mathrm{j}$ son file. In the third stage, a seeder script stores the JSON-file in the backend, thereby reflecting already existing versions (through versioning). After the seeder script has been executed, in the fourth stage, the API is ready to operate and communicate with the mobile applications.

\subsection{Medical Device Regulation}

In general, more and more countries require mHealth applications that are compliant with the medical device regulations (MDRs). When adhering to the MDRs, an important aspect constitutes the validation of the software, which shall ensure that a software is working correctly over time, i.e., always generating the same output for the same input. Therefore, the entire system has been validated on the basis of the IEC 62304 and IEC 82304 standards (medical device software/healthcare apps) as well as the GAMP 5 regulations (standard work of the pharmaceutical industry) [53]. These standards are the relevant parts of the medical device regulation (MDR) for implementing software that is compliant with the MDR. The basis for such development is a harmonized, risk-based approach developed from these regulations. Essentially, the regulations require the preparation of several document types, which finally serve as the basis for the validation of the software [53]. To be 
more precise, in the first step, the requirements of the application were collected in detail, in order to have a foundation to implement these requirements. The implementation took place in an agile process: design-programming — testing. As soon as all requirements have been implemented and tested in various development cycles, they were finally tested in a system test. The actual app development speed was decelerated by the relatively long requirement phase. However, this detailed analysis of the requirements and regulatory specifications led to an efficient error and problem identification and evaluation process. Therefore, during the further development course, potential error sources could be identified and eliminated rapidly.

\section{Collected Data}

At first, we listed the descriptions of the provided studies to illustrate the information introduced to participants:

- $\quad$ Study on Mental health for adults (18 years +): People's health is very important to us. Hence, we would like to ask you something: How are you feeling these days? While physical illness is prominent in news and the public, we are interested in the mood and possible stress factors that influence it. Moving freely, daily work routine, eating out, visiting grandparents, or meeting friends are important ingredients for us to feel good. Consequently, we would like to learn more about how you maintain social contacts, as these are important factors related to your well-being. In order to limit the effort for you as much as possible, we would like to automatically record your social contact via your smartphone (e.g., number and duration of calls, text messages). We would also like to automatically record your location at the time of the survey (GPS signal) in order to take regional differences into account. The data as well as your answers are stored completely anonymously, to profile you as a person is not possible. This first survey is a little more extensive and takes about $20 \mathrm{~min}$ - the follow-up surveys are only half of the length. By taking the time and participating in this survey you are a great help!

- $\quad$ Study on Mental health for adolescents (12 to 17 years): This scientific study investigates the burden on adolescents worldwide aged 12-17 years during the coronaviruspandemic. Understanding your struggles may help us physicians and scientists to develop strategies to better cope with the pandemic. Would you like to take part and help us? The first survey takes $15 \mathrm{~min}$. You may then take part in weekly follow-up surveys (5 $\mathrm{min})$. The study is completely anonymous. The study has been approved by an ethics committee and by a data protection official. No tracking is done by the app and only the data that you agree to will be transmitted anonymously. If you are unsure, whether you should take part, please talk to your parents about it. THANK YOU!

- $\quad$ Study on Physical health for adults (18 years +): Many things in our daily life have changed since the Corona crisis. This also includes more basic things such as our diet or our free time for example, how we do sports. These are factors that also have an influence on the development and course of diseases such as cardiovascular diseases. In the following questionnaire, we would thus like to learn more about how the Corona crisis affects your habits with a focus on factors that are related to the cardiovascular system. Filling in the questionnaire only takes about $15 \mathrm{~min}$. All data is collected anonymously. It is therefore not possible to relate any of the collected data with an individual person. Thank you for your support!

- $\quad$ Study on Recognizing stress for adults (18 years and up): Recognizing stress is important for your mental and physical health. That is why we invite you to fill out a short stress questionnaire once a week. People react very differently to stress. Therefore, we are also interested in factors that influence the experience of stress such as your age, your family status, your gender, and your smartphone usage (e.g., frequency of using communication services and social networks). The smartphone usage is recorded automatically to minimize the effort for you. Likewise, your whereabouts 
at the time of the survey are recorded automatically (GPS signal) in order to take regional differences in the stress experience into account. The data as well as your answers are stored completely anonymously. No conclusions can be drawn about the content of your social interactions or your person. Thank you very much for your effort and time in answering the questions about your perceived stress. You are a great help!

- Study on University Medicine Network: Compass project on the acceptance of pandemic apps (18 years and older): The variety of pandemic apps, apps that have been developed, for example, in hackathons to control COVID-19, shows the great potential that many experts see in them. But for apps to be effective in the pandemic, they must be used by many people. This applies not only to the Corona warning app, but also in particular to apps for assessing individual risk, for example in the case of certain pre-existing conditions. It is therefore necessary that such apps enjoy trust among the general population and that data can be analyzed together for medical research with the consent of the users. In COMPASS, scientists from a wide range of disciplines from university hospitals are joining forces with partners from science and industry in an interdisciplinary project to jointly develop a coordination and technology platform for pandemic apps. Help us with this survey so that pandemic apps can be developed even better and with broad acceptance and transparency in the future.

As of 11 May 2021, a total of 2802 users have registered in the five studies. They completed a total of 17,241 questionnaires. The study participants are between 12 and 120 years old (mean: 40.34, SD: 14.75) (the outlier with an age of 120 years likely did not enter the correct age). The ratio of Android to iOS users is about 4 to 1 across all studies. From 7290 verified users, only 2802 filled-out any questionnaire (38.4\%). High drop-out rates are a well-known issue in EMA studies [54]. From those 2802 users, 1476 filled out follow-up questionnaires (52.7\%). On average, each of those 1476 filled out 8.6 follow-up questionnaires. We provide more statistics about the collected data in Figure 9.

\begin{tabular}{|c|c|c|c|c|c|}
\hline Study name & Compass & Stress & Phys. $>18$ yrs & Psych. $>18$ yrs & Psych. $<18$ yrs \\
\hline $\mathrm{n}$ baselines & 314 & 371 & 1479 & 2141 & 190 \\
\hline $\mathrm{n}$ followups & not applicable & 1514 & 3588 & 7289 & 355 \\
\hline Females \% & $59.9 \%$ & $65.2 \%$ & $61.5 \%$ & $50.5 \%$ & $40.1 \%$ \\
\hline Male \% & $37.6 \%$ & $33.4 \%$ & $38.3 \%$ & $48.9 \%$ & $56.5 \%$ \\
\hline Diverse \% & $1.3 \%$ & $1.3 \%$ & $2.8 \%$ & $0.7 \%$ & $1.7 \%$ \\
\hline Not answered & $1.3 \%$ & $0.0 \%$ & $0.0 \%$ & $0.0 \%$ & $1.7 \%$ \\
\hline Age Distribution & & & $*$ & & \\
\hline$(12,13]$ & 0 & 0 & 0 & 0 & 21 \\
\hline$(13,14]$ & 0 & 0 & 0 & 0 & 29 \\
\hline$(14,15]$ & 0 & 0 & 0 & 0 & 33 \\
\hline$(15,16]$ & 0 & 0 & 0 & 0 & 46 \\
\hline$(16,17]$ & 1 & 0 & 0 & 0 & 48 \\
\hline$(17,30]$ & 79 & 99 & 97 & 531 & 0 \\
\hline$(30,40]$ & 71 & 100 & 82 & 566 & 0 \\
\hline$(40,50]$ & 65 & 79 & 64 & 453 & 0 \\
\hline$(50,60]$ & 49 & 62 & 69 & 380 & 0 \\
\hline$(60,70]$ & 31 & 29 & 38 & 171 & 0 \\
\hline$(70,80]$ & 5 & 2 & 8 & 37 & 0 \\
\hline$(80,120]$ & 1 & 0 & 0 & 4 & 0 \\
\hline Android & 258 & 303 & 315 & 1365 & 124 \\
\hline $\mathrm{iOS}$ & 44 & 68 & 43 & 777 & 31 \\
\hline Location perm. & $80.25 \%$ & $77.90 \%$ & $81.74 \%$ & $79.93 \%$ & $61.58 \%$ \\
\hline App perm. (Android) & $49.17 \%$ & $43.00 \%$ & $32.49 \%$ & $29.19 \%$ & $29.94 \%$ \\
\hline
\end{tabular}

Figure 9. Fact sheet for the five studies. A total of 17,241 questionnaires were completed by 11 May 2021. Note that demographic information was not available for the Adult Physical Health Questionnaire (Study "Phys. > 18 yrs") until the December 2020 update. 


\section{Discussion}

In this paper, we present the Corona Health app. It was conceived and implemented to enable observational studies that are able to explore aspects of the COVID-19 pandemic using smartphones. Based on the presented figures, we concluded that the app is frequently used and valuable data could be gathered. However, the data we collected likely contain a selection bias and the sample might not be representative of the general population [55]. A broader picture of society would have been desirable, but is challenging to achieve. Corona Health has been developed within 3 months and it is compliant with the Medical Device Regulation. To enable this, we have shown that Corona Health is based on existing technology as well as established procedures to manage the collaboration between the study domain experts and computer scientists. Practically, the following features constitute the key aspects in this context. They are summarized to show our experiences in developing apps such as Corona Health in a short period of time with many regulatory and organizational constraints:

- Our API can be flexibly used to manage various study types.

- Our apps can be flexibly tailored to the needs of researchers from the respective healthcare domain. Experience gained from previous projects, how such apps shall operate when applied large scale in real-life proved very valuable for the design procedure.

- Our proven and accepted content pipeline is able to flexibly add and change content between healthcare experts and computer scientists (see Section 3.6).

- We were able to find regulatory experts that are experienced in the MDR in the context of software engineering projects.

- Our general user journey of the app is accepted by participants.

Furthermore, for the feature to gather data about the used apps of Android participants, it can be concluded that it is a valuable addition. Participants mainly allow the measurement of this type of data and first results indicate that in the context of well-being and mental health app usage statistics might reveal interesting results. For example, a publication based on Corona Health data has found the following: Younger participants with higher use times tended to report less social well-being and higher loneliness, while the opposite effect was found for older adults [56].

To conclude, we constitute the framework that enables us to implement solutions such as Corona Health with its discussed features as a flexible and robust instrument. However, in practice, we also learned that many aspects are not covered so far. First, our questionnaire module is not able to dynamically navigate participants through a questionnaire. For example, if questions are not relevant for a specific participant, all dependent questions still have to be answered or inspected. Second, we identified scenarios in which our feedback module would require a more complex rule engine to cover all feedback scenarios. Third, a powerful visualization feature to enable participants viewing their own data directly within the app would be highly welcome. Finally, a feature should be offered that provides an extended onboarding procedure when the app is used for the first time (e.g., by the presentation of a video that explain the app). Altogether, Corona Health has now been running for almost one year and has revealed its usefulness for exploring aspects of the COVID-19 pandemic. Currently, we enhance our framework by new modules and improve the convenience with respect to content management. After that, other sensor measurements and more advanced interventions are planned (e.g., justin-time interventions). 
Author Contributions: Conceptualization, J.S., C.V., J.A., L.M., F.H., J.H., M.H., H.B., C.C., J.D. J.-S.E., F.A.E., H.G. G.H., P.H., D.J., T.P., M.R. (Marcel Romanos), W.S., Y.T. and R.P.; data curation, J.A., M.S. (Michael Stach) and R.P.; formal analysis, J.A. and R.P.; funding acquisition, R.P.; investigation, J.S., C.V., L.M., M.H., H.B., C.C., J.-S.E., F.A.E., P.H., D.J., H.A.K., D.K., B.L., T.P., M.R. (Marcel Romanos), W.S., S.S., Y.T. and R.P.; methodology, J.S., C.V., J.A., L.M., F.H., J.H., H.B., C.C., J.D., J.-S.E., F.A.E., G.H., P.H., D.J., D.K., B.L., P.M., T.P., M.R. (Marcel Romanos), W.S., S.S., Y.T. and R.P.; project administration, J.S., C.V., L.M., M.H., H.B., C.C., J.-S.E., F.A.E., P.H., D.J., B.L., T.P., M.R. (Manfred Reichert), M.R. (Marcel Romanos), W.S., S.S., Y.T. and R.P.; resources, H.G., P.H., H.A.K., M.R. (Manfred Reichert), M.R. (Marcel Romanos), W.S. and R.P.; software, J.S., C.V., L.M., F.H., J.H., H.G. and R.P.; supervision, J.S., H.B., C.C., J.-S.E., P.H., D.J., M.R. (Manfred Reichert), Y.T. and R.P.; validation, J.S., L.M., M.H., W.S. and R.P.; visualization, J.A., M.S. (Michael Stach) and M.S. (Marc Schickler); writing - original draft preparation, F.B., J.S., J.A., L.M., M.S. (Michael Stach) and R.P.; writing-review and editing, F.B., J.S., C.V., J.A., L.M., F.H., J.H., M.H., M.S. (Michael Stach), M.S. (Marc Schickler), H.B., C.C., J.D., L.D., J.-S.E., F.A.E., H.G., G.H., P.H., D.J., H.A.K., D.K., B.L., P.M., T.P., M.R. (Manfred Reichert), M.R. (Marcel Romanos), W.S., S.S., Y.T., M.W. and R.P. All authors have read and agreed to the published version of the manuscript.

Funding: F.B., L.M., F.H., J.H., M.S. (Michael Stach), C.C., P.H., D.K., B.L., W.S., and R.P. are supported by grants in the project COMPASS. P.H. and R.P. are supported by a grant in the project NAPKON. The COMPASS and NAPKON projects are part of the German COVID-19 Research Network of University Medicine ("Netzwerk Universitätsmedizin"), funded by the German Federal Ministry of Education and Research (funding reference 01KX2021). L.D. is supported by a grant from the German Research Foundation (TRR 265, Project A02, 428318753) and by the Federal Ministry for Education and Research, Germany (FKZ: 01EO1501). G.H. is supported by a grant from the German Research Foundation (HE 4566/5-1). This publication was supported by the Open Access Publication Fund of the University of Wuerzburg.

Institutional Review Board Statement: The Corona Health app study was conducted in accordance with the German medical products law. The data protection officer and the ethics committee of the University of Würzburg, Germany, approved the study (No. 130/20-me). The procedures used in this study adhere to the tenets of the Declaration of Helsinki.

Informed Consent Statement: Informed consent was obtained from all subjects involved in the study.

Data Availability Statement: The data presented in this study are available on request from the corresponding author. The data are not publicly available because participants' informed consent did not cover public deposition of data.

Acknowledgments: We are grateful for the script programming provided by Leoni Holl and Klaus Kammerer and for the support with the visual design of the app provided by Michael Schultz.

Conflicts of Interest: J.D. is an investigator in the EU-Horizon-funded Predict Study of P1Vital and Co-Applicant with BioVariance in the InDepth Study funded by the Bavarian State Government.

\section{References}

1. Zhang, Y.; Ma, Z.F. Impact of the COVID-19 Pandemic on Mental Health and Quality of Life among Local Residents in Liaoning Province, China: A Cross-Sectional Study. Int. J. Environ. Res. Public Health 2020, 17, 2381. [CrossRef]

2. Wang, C.; Pan, R.; Wan, X.; Tan, Y.; Xu, L.; Ho, C.S.; Ho, R.C. Immediate Psychological Responses and Associated Factors during the Initial Stage of the 2019 Coronavirus Disease (COVID-19) Epidemic among the General Population in China. Int. J. Environ. Res. Public Health 2020, 17, 1729. [CrossRef] [PubMed]

3. Gualano, M.R.; Lo Moro, G.; Voglino, G.; Bert, F.; Siliquini, R. Effects of Covid-19 Lockdown on Mental Health and Sleep Disturbances in Italy. Int. J. Environ. Res. Public Health 2020, 17, 4779. [CrossRef] [PubMed]

4. Xiong, J.; Lipsitz, O.; Nasri, F.; Lui, L.M.W.; Gill, H.; Phan, L.; Chen-Li, D.; Iacobucci, M.; Ho, R.; Majeed, A.; et al. Impact of COVID-19 Pandemic on Mental Health in the General Population: A Systematic Review. J. Affect. Disord. 2020, 277, 55-64. [CrossRef] [PubMed]

5. Gloster, A.T.; Lamnisos, D.; Lubenko, J.; Presti, G.; Squatrito, V.; Constantinou, M.; Nicolaou, C.; Papacostas, S.; Aydın, G.; Chong, Y.Y.; et al. Impact of COVID-19 Pandemic on Mental Health: An International Study. PLoS ONE 2020, 15, e0244809. [CrossRef] [PubMed] 
6. Limcaoco, R.S.G.; Mateos, E.M.; Fernández, J.M.; Roncero, C. Anxiety, Worry and Perceived Stress in the World Due to the COVID-19 Pandemic, March 2020. Preliminary Results. medRxiv 2020. [CrossRef]

7. Pieh, C.; Budimir, S.; Probst, T. The Effect of Age, Gender, Income, Work, and Physical Activity on Mental Health during Coronavirus Disease (COVID-19) Lockdown in Austria. J. Psychosom. Res. 2020, 136, 110186. [CrossRef] [PubMed]

8. Pierce, M.; McManus, S.; Hope, H.; Hotopf, M.; Ford, T.; Hatch, S.L.; John, A.; Kontopantelis, E.; Webb, R.T.; Wessely, S.; et al. Mental Health Responses to the COVID-19 Pandemic: A Latent Class Trajectory Analysis Using Longitudinal UK Data. Lancet Psychiatry 2021. [CrossRef]

9. Pieh, C.; Budimir, S.; Delgadillo, J.; Barkham, M.; Fontaine, J.R.J.; Probst, T. Mental Health During COVID-19 Lockdown in the United Kingdom. Psychosom. Med. 2021, 83, 328-337. [CrossRef]

10. Dale, R.; Budimir, S.; Probst, T.; Stippl, P.; Pieh, C. Mental Health during the COVID-19 Lockdown over the Christmas Period in Austria and the Effects of Sociodemographic and Lifestyle Factors. Int. J. Environ. Res. Public Health 2021, 18, 3679. [CrossRef]

11. Budimir, S.; Pieh, C.; Dale, R.; Probst, T. Severe Mental Health Symptoms during COVID-19: A Comparison of the United Kingdom and Austria. Healthcare 2021, 9, 191. [CrossRef]

12. Ferreira, D.; Kostakos, V.; Dey, A.K. AWARE: Mobile Context Instrumentation Framework. Front. ICT 2015, 2. [CrossRef]

13. Xiong, H.; Huang, Y.; Barnes, L.E.; Gerber, M.S. Sensus: A Cross-Platform, General-Purpose System for Mobile Crowdsensing in Human-Subject Studies. In Proceedings of the 2016 ACM International Joint Conference on Pervasive and Ubiquitous Computing (UbiComp 2016), Heidelberg, Germany, 12-16 September 2016; pp. 415-426. [CrossRef]

14. Schobel, J.; Probst, T.; Reichert, M.; Schickler, M.; Pryss, R. Enabling sophisticated lifecycle support for mobile healthcare data collection applications. IEEE Access 2019, 7, 61204-61217. [CrossRef]

15. Schobel, J.; Pryss, R.; Schickler, M.; Ruf-Leuschner, M.; Elbert, T.; Reichert, M. End-user programming of mobile services: empowering domain experts to implement mobile data collection applications. In Proceedings of the 2016 IEEE International Conference on Mobile Services (MS), San Francisco, CA, USA, 27 June-2 July 2016; pp. 1-8.

16. Kumar, D.; Jeuris, S.; Bardram, J.E.; Dragoni, N. Mobile and Wearable Sensing Frameworks for mHealth Studies and Applications: A Systematic Review. ACM Trans. Comput. Healthc. 2020, 2, 8:1-8:28. [CrossRef]

17. Hamel, M.B.; Cortez, N.G.; Cohen, I.G.; Kesselheim, A.S. FDA regulation of mobile health technologies. N. Engl. J. Med. 2014, 371,372 .

18. Jogova, M.; Shaw, J.; Jamieson, T. The regulatory challenge of mobile health: Lessons for Canada. Healthc. Policy 2019, 14, 19. [CrossRef] [PubMed]

19. Vogel, C.; Pryss, R.; Schobel, J.; Schlee, W.; Beierle, F. Developing Apps for Researching the COVID-19 Pandemic with the TrackYourHealth Platform. In Proceedings of the 2021 IEEE/ACM 8th International Conference on Mobile Software Engineering and Systems (MobileSoft), Pittsburgh, PA, USA, 17-19 May 2021; pp. 65-68. [CrossRef]

20. Pryss, R.; Schlee, W.; Langguth, B.; Reichert, M. Mobile crowdsensing services for tinnitus assessment and patient feedback. In Proceedings of the 2017 IEEE International Conference on AI \& Mobile Services (AIMS), Honolulu, HI, USA, 25-30 June 2017; pp. 22-29.

21. Pryss, R.; John, D.; Schlee, W.; Schlotz, W.; Schobel, J.; Kraft, R.; Spiliopoulou, M.; Langguth, B.; Reichert, M.; O’Rourke, T.; et al. Exploring the time trend of stress levels while using the Crowdsensing Mobile health platform, TrackYourStress, and the influence of perceived stress reactivity: Ecological momentary assessment pilot study. JMIR mHealth uHealth 2019, 7, e13978. [CrossRef]

22. Unnikrishnan, V.; Shah, Y.; Schleicher, M.; Strandzheva, M.; Dimitrov, P.; Velikova, D.; Pryss, R.; Schobel, J.; Schlee, W.; Spiliopoulou, M. Predicting the Health Condition of mHealth App Users with Large Differences in the Number of Recorded Observations-Where to Learn from? In International Conference on Discovery Science; Springer: Berlin/Heidelberg, Germany, 2020; pp. 659-673.

23. Stone, A.A.; Shiffman, S. Ecological Momentary Assessment (EMA) in Behavioral Medicine. Ann. Behav. Med. 1994, 16, 199-202. [CrossRef]

24. Larson, R.; Csikszentmihalyi, M. The Experience Sampling Method. In Flow and the Foundations of Positive Psychology: The Collected Works of Mihaly Csikszentmihalyi; Reis, H., Ed.; Volume Flow and the Foundations of Positive Psychology; Springer: Dordrecht, The Netherlands, 2014; pp. 41-56. [CrossRef]

25. van Berkel, N.; Ferreira, D.; Kostakos, V. The Experience Sampling Method on Mobile Devices. ACM Comput. Surv. 2017, 50, 93:1-93:40. [CrossRef]

26. Pryss, R.; Probst, T.; Schlee, W.; Schobel, J.; Langguth, B.; Neff, P.; Spiliopoulou, M.; Reichert, M. Prospective Crowdsensing versus Retrospective Ratings of Tinnitus Variability and Tinnitus-Stress Associations Based on the TrackYourTinnitus Mobile Platform. Int. J. Data Sci. Anal. 2019, 8, 327-338. [CrossRef]

27. Beierle, F.; Tran, V.T.; Allemand, M.; Neff, P.; Schlee, W.; Probst, T.; Pryss, R.; Zimmermann, J. Context Data Categories and Privacy Model for Mobile Data Collection Apps. Procedia Comput. Sci. 2018, 134, 18-25. [CrossRef]

28. Burke, J.; Estrin, D.; Hansen, M.; Parker, A.; Ramanathan, N.; Reddy, S.; Srivastava, M.B. Participatory Sensing. In Workshop on World-Sensor-Web (WSW'06): Mobile Device Centric Sensor Networks and Applications; ACM: New York, NY, USA, 2006 ; pp. 117-134. 
29. Lane, N.D.; Miluzzo, E.; Lu, H.; Peebles, D.; Choudhury, T.; Campbell, A.T. A Survey of Mobile Phone Sensing. IEEE Commun. Mag. 2010, 48, 140-150. [CrossRef]

30. Ganti, R.K.; Ye, F.; Lei, H. Mobile Crowdsensing: Current State and Future Challenges. IEEE Commun. Mag. 2011, 49, 32-39. [CrossRef]

31. Kraft, R.; Schlee, W.; Stach, M.; Reichert, M.; Langguth, B.; Baumeister, H.; Probst, T.; Hannemann, R.; Pryss, R. Combining mobile crowdsensing and ecological momentary assessments in the healthcare domain. Front. Neurosci. 2020, 14, 164. [CrossRef] [PubMed]

32. Boubiche, D.E.; Imran, M.; Maqsood, A.; Shoaib, M. Mobile Crowd Sensing-Taxonomy, Applications, Challenges, and Solutions. Comput. Hum. Behav. 2019, 101, 352-370. [CrossRef]

33. Pryss, R. Mobile crowdsensing in healthcare scenarios: Taxonomy, conceptual pillars, smart mobile crowdsensing services. In Digital Phenotyping and Mobile Sensing; Springer: Cham, Switzerland, 2019; pp. 221-234.

34. Baumeister, H.; Montag, C. (Eds.) Digital Phenotyping and Mobile Sensing: New Developments in Psychoinformatics; Studies in Neuroscience, Psychology and Behavioral Economics; Springer: Cham, Switzerland, 2019. [CrossRef]

35. Insel, T.R. Digital Phenotyping: Technology for a New Science of Behavior. JAMA 2017, 318, 1215-1216.10.1001/jama.2017.11295. [CrossRef] [PubMed]

36. Jain, S.H.; Powers, B.W.; Hawkins, J.B.; Brownstein, J.S. The Digital Phenotype. Nat. Biotechnol. 2015, 33, 462-463. [CrossRef] [PubMed]

37. Montag, C.; Sindermann, C.; Baumeister, H. Digital Phenotyping in Psychological and Medical Sciences: A Reflection about Necessary Prerequisites to Reduce Harm and Increase Benefits. Curr. Opin. Psychol. 2020, 36, 19-24. [CrossRef] [PubMed]

38. Onnela, J.P. Opportunities and Challenges in the Collection and Analysis of Digital Phenotyping Data. Neuropsychopharmacology 2021, 46, 45-54. [CrossRef]

39. Beierle, F. Integrating Psychoinformatics with Ubiquitous Social Networking: Advanced Mobile-Sensing Concepts and Applications; Springer: Cham, Switzerland, 2021. [CrossRef]

40. Montag, C.; Baumeister, H.; Kannen, C.; Sariyska, R.; Meßner, E.M.; Brand, M. Concept, Possibilities and Pilot-Testing of a New Smartphone Application for the Social and Life Sciences to Study Human Behavior Including Validation Data from Personality Psychology. J 2019, 2, 102-115. [CrossRef]

41. Beierle, F.; Matz, S.C.; Allemand, M. Mobile Sensing in Personality Science. In Mobile Sensing in Psychology: Methods and Applications; Mehl, M.R., Wrzus, C., Eid, M., Harari, G., Priemer, U.E., Eds.; Guilford Press: New York, NY, USA, 2021; in press.

42. Rooksby, J.; Morrison, A.; Murray-Rust, D. Student Perspectives on Digital Phenotyping: The Acceptability of Using Smartphone Data to Assess Mental Health. In Proceedings of the 2019 CHI Conference on Human Factors in Computing Systems, Glasgow, UK, 4-9 May 2019; pp. 425:1-425:14. [CrossRef]

43. Zulueta, J.; Piscitello, A.; Rasic, M.; Easter, R.; Babu, P.; Langenecker, S.A.; McInnis, M.; Ajilore, O.; Nelson, P.C.; Ryan, K.; et al. Predicting Mood Disturbance Severity with Mobile Phone Keystroke Metadata: A BiAffect Digital Phenotyping Study. J. Med. Internet Res. 2018, 20, e241. [CrossRef] [PubMed]

44. Beierle, F.; Tran, V.T.; Allemand, M.; Neff, P.; Schlee, W.; Probst, T.; Zimmermann, J.; Pryss, R. What Data Are Smartphone Users Willing to Share with Researchers? J. Ambient. Intell. Humaniz. Comput. 2020, 11, 2277-2289. [CrossRef]

45. Borra, S. COVID-19 Apps: Privacy and Security Concerns. In Intelligent Systems and Methods to Combat Covid-19; Joshi, A., Dey, N., Santosh, K.C., Eds.; SpringerBriefs in Applied Sciences and Technology; Springer: Singapore, 2020; pp. 11-17. [CrossRef]

46. Ahmed, N.; Michelin, R.A.; Xue, W.; Ruj, S.; Malaney, R.; Kanhere, S.S.; Seneviratne, A.; Hu, W.; Janicke, H.; Jha, S.K. A Survey of COVID-19 Contact Tracing Apps. IEEE Access 2020, 8, 134577-134601. [CrossRef]

47. Pryss, R.; Schobel, J.; Reichert, M. Requirements for a Flexible and Generic API Enabling Mobile Crowdsensing mHealth Applications. In Proceedings of the 2018 4th International Workshop on Requirements Engineering for Self-Adaptive, Collaborative, and Cyber Physical Systems (RESACS), Banff, AB, Canada, 20-20 August 2018; pp. 24-31. [CrossRef]

48. Pryss, R.; Schlee, W.; Hoppenstedt, B.; Reichert, M.; Spiliopoulou, M.; Langguth, B.; Breitmayer, M.; Probst, T. Applying Machine Learning to Daily-Life Data From the TrackYourTinnitus Mobile Health Crowdsensing Platform to Predict the Mobile Operating System Used With High Accuracy: Longitudinal Observational Study. J. Med. Internet Res. 2020, 22, e15547. [CrossRef]

49. Canzian, L.; Musolesi, M. Trajectories of Depression: Unobtrusive Monitoring of Depressive States by Means of Smartphone Mobility Traces Analysis. In Proceedings of the 2015 ACM International Joint Conference on Pervasive and Ubiquitous Computing, Osaka, Japan, 7-11 September 2015; pp. 1293-1304. [CrossRef]

50. Heller, A.S.; Shi, T.C.; Ezie, C.E.C.; Reneau, T.R.; Baez, L.M.; Gibbons, C.J.; Hartley, C.A. Association between Real-World Experiential Diversity and Positive Affect Relates to Hippocampal-Striatal Functional Connectivity. Nat. Neurosci. 2020, 23, 800-804. [CrossRef]

51. Beierle, F.; Probst, T.; Allemand, M.; Zimmermann, J.; Pryss, R.; Neff, P.; Schlee, W.; Stieger, S.; Budimir, S. Frequency and Duration of Daily Smartphone Usage in Relation to Personality Traits. Digit. Psychol. 2020, 1, 20-28. [CrossRef]

52. Gründahl, M.; Deckert, J.; Hein, G. Three questions to consider before applying ecological momentary interventions (EMI) in psychiatry. Front. Psychiatry 2020, 11, 333. [CrossRef]

53. Holfelder, M.; Mulansky, L.; Schlee, W.; Baumeister, H.; Schobel, J.; Greger, H.; Hoff, A.; Pryss, R. Medical Device Regulation Efforts for mHealth Apps - An Experience Report of Corona Check and Corona Health. arXiv 2021, arXiv:2104.13635. 
54. Seifert, A.; Hofer, M.; Allemand, M. Mobile Data Collection: Smart, but Not (Yet) Smart Enough. Front. Neurosci. $2018,12$. [CrossRef] [PubMed]

55. Hargittai, E. Potential Biases in Big Data: Omitted Voices on Social Media. Soc. Sci. Comput. Rev. 2020, 38, 10-24. [CrossRef]

56. Wetzel, B.; Pryss, R.; Baumeister, H.; Edler, J.S.; Gonçalves, A.S.O.; Cohrdes, C. “How Come You Don't Call Me?" Smartphone Communication App Usage as an Indicator of Loneliness and Social Well-Being across the Adult Lifespan during the COVID-19 Pandemic. Int. J. Environ. Res. Public Health 2021, 18, 6212. [CrossRef] [PubMed] 